\title{
Steps in the hysteresis loops of a high-spin molecule
}

\author{
Jonathan R. Friedman and M. P. Sarachik \\ Department of Physics, The City College of New York, New York, New York 10031 \\ J. Tejada \\ Facultat de Física, Universitat de Barcelona, 08028 Barcelona, Spain
}

J. Maciejewski and R. Ziolo

Wilson Research Center, Xerox Corporation, Webster, New York 14580

\begin{abstract}
We report the first observation of steps in the hysteresis loop of a high-spin molecular magnet. We propose that the steps, which occur every $0.46 \mathrm{~T}$, are due to thermally assisted resonant tunneling between different quantum spin states. Magnetic relaxation increases dramatically when the field is in the neighborhood of a step. A simple model accounts for the observations and predicts a value for the anisotropy barrier consistent with that inferred from the superparamagnetic blocking temperature. (C) 1996 American Institute of Physics. [S0021-8979(96)41008-0]
\end{abstract}

\section{INTRODUCTION}

The large-spin molecule $\mathrm{Mn}_{12} \mathrm{O}_{12}\left(\mathrm{CH}_{3} \mathrm{COO}\right)_{16}\left(\mathrm{H}_{2} \mathrm{O}\right)_{4}$, has been the subject of much experimental ${ }^{1-5,10}$ and theoretical ${ }^{6,7}$ work since it was first synthesized by $\mathrm{Lis}^{8}$ in 1980. This molecule (often referred to as $\mathrm{Mn}_{12}$ ) contains four $\mathrm{Mn}^{+4} \quad(S=3 / 2)$ ions and eight $\mathrm{Mn}^{+3}(S=2)$ ions. Experiments ${ }^{1}$ indicate that it has an $S=10$ ground state. These molecules crystallize into a tetragonal lattice in which magnetic interactions between molecules are thought to be negligible. ${ }^{3}$ All experimental work ${ }^{1-5,10}$ to date on this system indicates that it has a large magnetocrystalline anisotropy. Superparamagneticlike behavior has been reported. ${ }^{1-4}$ Below a blocking temperature of $\sim 3 \mathrm{~K}$, hysteresis is observed $^{3,5}$ and slow exponential relaxation of the magnetization has been found ${ }^{3}$ with relaxation times that obey an Arrhenius law, $\tau=\tau_{0} e^{\Delta E / k_{B} T}$, down to $2.1 \mathrm{~K}$; studies ${ }^{4,10}$ at temperatures down to $175 \mathrm{mK}$ show deviations from this form that have been interpreted as possible signs of temperature-independent quantum tunneling.

In this article, we report the observation of steps in the hysteresis loops of a powdered sample of $\mathrm{Mn}_{12}$. We suggest that the steps are manifestations of thermally assisted, fieldtuned resonant tunneling through the molecule's anisotropy barrier. We show that the relaxation rate increases dramatically when the applied field is tuned to a field at which one of the steps occurs.

\section{EXPERIMENTS AND RESULTS}

The sample was prepared following the published procedure $^{8}$ and was then ground into a powder consisting of submicron-sized crystallites. The powdered sample was mixed into Stycast 1266 and allowed to set in a field of $5.5 \mathrm{~T}$ at $300 \mathrm{~K}$. This served to orient the crystallites suspended in the epoxy such that their easy axes aligned with the field due to the anisotropy of the susceptibility tensor. No corrections were made for the diamagnetism of the epoxy, which was considered negligible. The dc magnetization measurements were taken at temperatures between 1.7 and $15 \mathrm{~K}$ with a Quantum Design MPMS5 magnetometer equipped with a 5.5-T superconducting magnet.

Figure 1 shows the hysteresis loops taken with the field applied along the easy axis of the oriented sample at six different temperatures from 1.7 to $2.8 \mathrm{~K}$, as indicated. The steps in the loops are clearly visible. In contrast, no steps were seen in a control sample prepared in the absence of a field. It is noteworthy that after the system is saturated and the field is reduced back to zero field, the curve is temperature independent and no steps are apparent. The steps occur only at specific values of magnetic field. The inset to Fig. 1 shows the field at which a step occurs plotted versus step number, where the steps are labeled by integers and the fields plotted are the points in the hysteresis curves where the slope, $d M / d H$, is maximal. The excellent linear fit indicates that a step occurs at approximately every $0.46 \mathrm{~T}$. If data from all temperatures are used, then a total of seven steps, including the one at zero field, are observed and it is quite possible that more would be seen at lower temperatures. We note that as temperature is lowered, steps that were apparent at higher temperatures seem to disappear. These steps can still be observed, however, if the rate at which the field is swept is

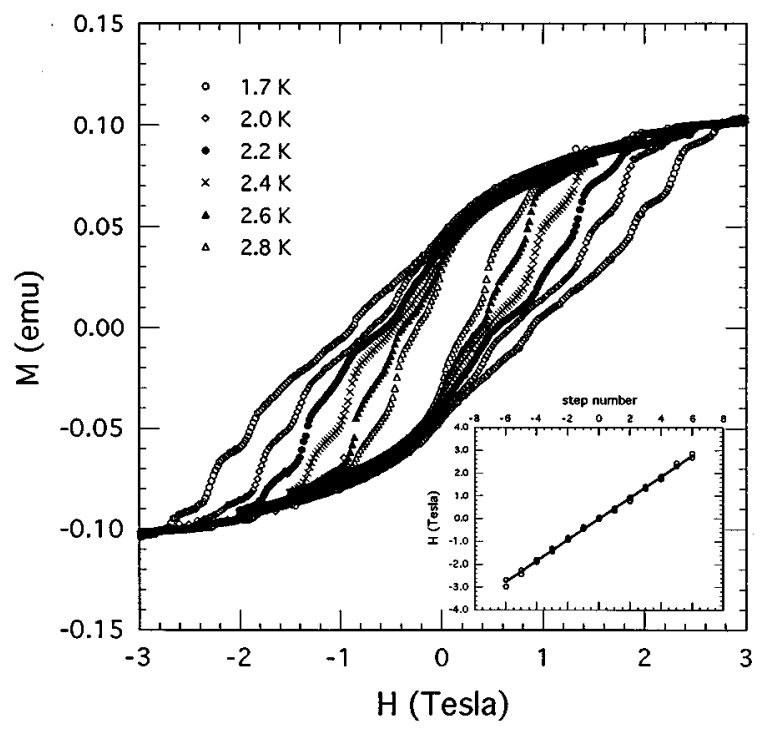

FIG. 1. Hysteresis loops of $\mathrm{Mn}_{12}$ for six different temperatures, as shown. The inset shows the fields at which steps occur as a function of step number. The straight line is a least-squares fit. 


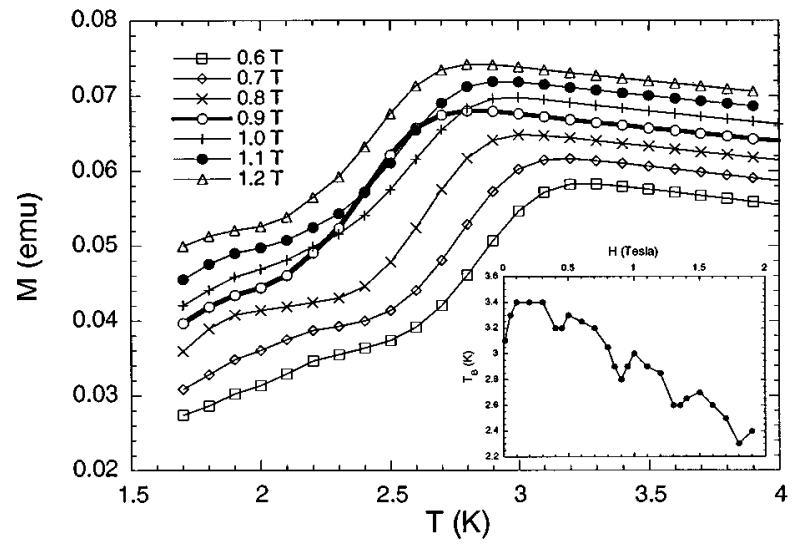

FIG. 2. Zero-field-cooled data for seven different measuring fields. The data for $0.9 \mathrm{~T}$ (bold line) is anomalous, shifting abruptly toward lower temperatures. The inset shows the superparamagnetic blocking temperature, $T_{B}$, as a function of magnetic field. The anomalous dips occur at approximately the same fields as the steps in the hysteresis loops.

reduced sufficiently. Similar steps have been observed ${ }^{9}$ at 0.6 $\mathrm{K}$ in $\left[\mathrm{Fe}(\mathrm{OMe})_{2}\left(\mathrm{O}_{2} \mathrm{CCH}_{2} \mathrm{C} 1\right)\right]_{10}$, a molecular paramagnet comprised of a ring of $\mathrm{Fe}^{3+}$ ions which order antiferromagnetically and exhibit no hysteretic effects. Our results appear to be the first observation of such steps in a hysteretic molecular system.

In Fig. 2 we show magnetization data of the $\mathrm{Mn}_{12}$ sample after it was cooled from 5 to $1.7 \mathrm{~K}$ in zero field and then measured in an applied field as the temperature was raised. Data for seven different measuring fields, as indicated, are shown. (Some curves at other fields were omitted for clarity.) As expected for a superparamagnetic system, the blocking temperature $T_{B}$ (the temperature of the curve's maximum) decreases systematically as the measuring field is raised. However, the curve at $0.9 \mathrm{~T}$ shifts abruptly toward lower temperatures. In the inset, we show the blocking temperature as a function of magnetic field from 0.01 to $1.9 \mathrm{~T}$. Superimposed on the monatonic decrease of these temperatures with increasing field are several sharp dips. These dips occur at approximately the same fields as the steps in the hysteresis loops of Fig. 1. Ignoring these anomalous dips, we extrapolate the zero-field blocking temperature to be $\sim 3.5 \mathrm{~K}$. This corresponds to an energy barrier, $\Delta E$, of $45 \mathrm{~cm}^{-1}$.

An interesting feature of the zero-field-cooled curves is that some of them cross: in Fig. 2, one can see that within a certain temperature range the magnetization attains a higher value at 0.9 than at $1 \mathrm{~T}$ and sometimes even $1.1 \mathrm{~T}$. This implies that the relaxation rate of the system must be significantly faster at the lower field value than at the higher ones. Direct evidence for this is provided in Fig. 3, where we have plotted the difference between the magnetization and its asymptotic value, $M_{0}$, as a function of time on a semilogarithmic plot. The curves show the relaxation of the magnetization after the sample was cooled to $2.4 \mathrm{~K}$ in zero field and then exposed to a field of 0.9 or $0.95 \mathrm{~T}$, as marked. There is a dramatically faster relaxation rate at 0.9 than at $0.95 \mathrm{~T}$. During the initial $\sim 2000 \mathrm{~s}$, the magnetization decays faster than exponentially. The data for $t \geqslant 2000 \mathrm{~s}$ can be fitted very well to an exponential, $M=M_{0}\left(1-e^{-\left(t-t_{0}\right) / \tau}\right)$, as shown by

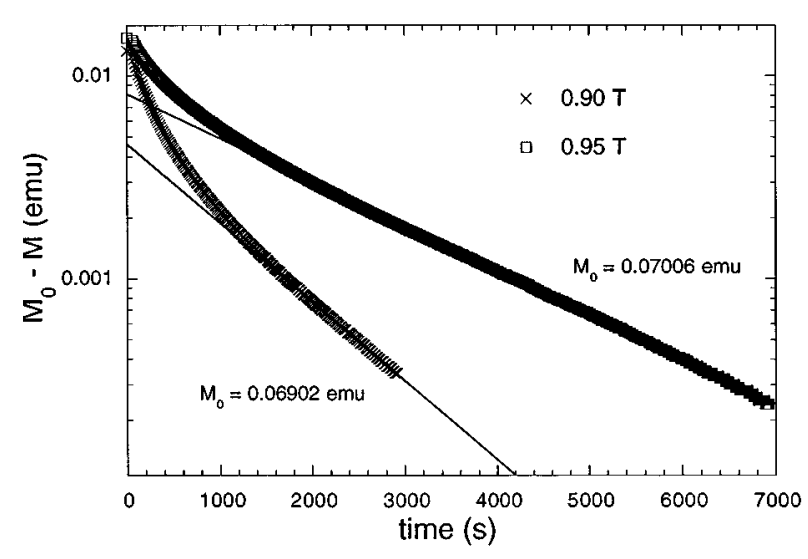

FIG. 3. Magnetic relaxation at $2.4 \mathrm{~K}$ plotted on a semilogarithmic scale. After cooling in zero field, a field of 0.9 or $0.95 \mathrm{~T}$ was applied, as indicated. $M_{0}$ is the asymptotic value of the magnetization; the straight lines indicate exponential behavior for $t>2000 \mathrm{~s}$.

the straight lines in Fig. 3. The fit yields time constants, $\tau$, of 1048 and $2072 \mathrm{~s}$ at 0.9 and $0.95 \mathrm{~T}$, respectively.

\section{DISCUSSION}

We suggest that the observed steps are due to thermally assisted resonant tunneling between quantum spin states in the $\mathrm{Mn}_{12}$ system. In zero field, the molecule has two degenerate ground states, corresponding to the spin being parallel $(m=S)$ or antiparallel $(m=-S)$ to the easy axis. An anisotropy barrier separates the states and a magnetic field breaks the symmetry, making one state a true ground state and the other a metastable ground state. This model is illustrated in Fig. 4. When an excited level in the right well is resonant with the metastable $m=S$ ground state on the left, transitions across the barrier are induced. Once the system has crossed the barrier, there is presumably a rapid spontaneous decay from the excited state to the ground state. In this way the metastable state is depleted and the system enters the $m=-S$ state.

The simplest Hamiltonian for this system is

$$
\mathscr{H}=-D \mathbf{S}_{z}^{2}-g \mu_{B} \mathbf{S} \cdot \mathbf{H},
$$

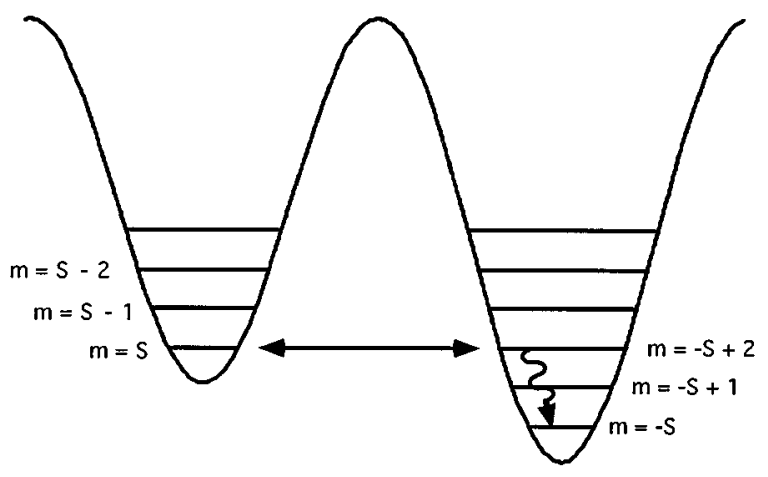

FIG. 4. A schematic representation of the resonant tunneling mechanism proposed to explain the observations. Tunneling from the metastable state $m=S$ to an excited state $m=-S+n$ is followed by a rapid spontaneous decay into the ground state. 
where $D$ represents the anisotropy energy that breaks the zero-field Zeeman degeneracy. If the field is applied along the easy axis, then the eigenstates of this Hamiltonian are $|S, m\rangle$. The first term in Eq. (1) implies that the ground state will be $m= \pm S$, as expected; the sign depends on the direction of the field. For clarity, we take $|S, S\rangle$ as the initial state of the system. We propose that a step occurs when the energy of this state is equal to the energy of a state on the other side of the anisotropy barrier. A simple calculation reveals that the field at which the state $|S, S\rangle$ crosses the state $|S,-S+n\rangle$, is

$$
H_{S,-S+n}=\frac{-D n}{g \mu_{B}} .
$$

Thus, steps occur at even intervals of field, as observed. Interestingly, when the field is tuned such that the metastable ground state in the left well is resonant with a state in the right well, all of the excited states in the left well are also resonant with states on the other side of the barrier. Thus, a multiple resonance is set up whenever the field is tuned to a step. Using the fact that a step occurs every $0.46 \mathrm{~T}$, we find $D / g=0.21 \mathrm{~cm}^{-1}$, which is consistent with the published values $^{1}$ of $D \sim 0.5 \mathrm{~cm}^{-1}$ and $g \sim 1.9 \mathrm{~cm}^{-1}$. As a further check of this model, we can estimate the size of the anisotropy barrier: at zero field, the barrier is $g(D / g) S^{2} \sim 41 \mathrm{~cm}^{-1}$, consistent with the estimate of $45 \mathrm{~cm}^{-1}$ obtained above from the blocking temperature data. Given that $S=10$ in this system, there should be exactly 21 steps $(n=0-20)$, the last corresponding to the elimination of the barrier. Measurements at lower temperatures are needed to observe additional steps.

As noted above, as temperature is reduced, any given step becomes less prominent and the transition rate decreases rapidly. This implies that the resonant tunneling mechanism responsible for the steps is thermally assisted. It is possible that acoustic phonons provide the angular momentum necessary for the transition between different spin states. As temperature is lowered, the population of phonons (or other excitations) drops and the transition rate decreases. More work is needed to understand precisely what role thermal excitations play in this resonant process.

We would like to stress that the above model of fieldtuned, thermally assisted resonant tunneling out of a metastable spin state explains many of the observations presented herein. When the field is reduced from saturation, no steps are seen because the system is already in the true ground state $m=S$. It is only when the field is reduced to near zero or reversed that the state becomes metastable allowing resonant transitions and the corresponding steps. The higher- numbered steps have progressively faster magnetic relaxation times since the tunnel barrier is lowered by the applied field.

In summary, we have found steps in the hysteresis curves of $\mathrm{Mn}_{12} \mathrm{O}_{12}\left(\mathrm{CH}_{3} \mathrm{COO}\right)_{16}\left(\mathrm{H}_{2} \mathrm{O}\right)_{4}$ and propose that they result from thermally assisted resonant transitions between spin states. The magnetic relaxation rate of the system increases dramatically when the field is tuned to a step; that is, when there is a level crossing. A simple model has been proposed that accounts for the even interval of field between neighboring steps and is in quantitative agreement with measurements of the anisotropy barrier.

Note added in proof: Since we submitted this manuscript, two papers ${ }^{11,12}$ have appeared on $\mathrm{Mn}_{12}$, reporting anomalously fast relaxation rates at zero field ${ }^{11,12}$ and 0.3 $\mathrm{T} .{ }^{12}$ Novak and Sessoli ${ }^{12}$ suggest that these results are due to thermally assisted resonant tunneling. A similar suggestion has been made previously by Barbara et al. ${ }^{10}$ to explain the zero-field anomaly.

\section{ACKNOWLEDGMENTS}

We would like to thank Dariusz Przybylski for assistance in preparing some control samples and Eugene Chudnovsky, Snezana Bogdanovich, and Dana Calistru for useful conversations. This work was supported by the Air Force Office of Scientific Research under Grant No. F49620-92-J-0190.

${ }^{1}$ A. Caneschi, D. Gatteschi, R. Sessoli, A. L. Barra, L. C. Brunel, and M. Guillot, J. Am. Chem. Soc. 113, 5873 (1991).

${ }^{2}$ R. Sessoli, H.-L. Tsai, A. R. Schake, S. Wang, J. B. Vincent, K. Folting, D. Gatteschi, G. Christou, and D. N. Hendrickson, J. Am. Chem. Soc. 115, 1804 (1993).

${ }^{3}$ R. Sessoli, D. Gatteschi, A. Caneschi, and M. A. Novak, Nature 365, 141 (1993).

${ }^{4}$ C. Paulsen, J.-G. Park, B. Barbara, R. Sessoli, and A. Caneschi, J. Magn. Magn. Mater. 140-144, 379 (1995).

${ }^{5}$ C. Paulsen, J.-G. Park, B. Barbara, R. Sessoli, and A. Caneschi, J. Magn. Magn. Mater. 140-144, 1891 (1995).

${ }^{6}$ J. Villain, F. Hartman-Boutron, R. Sessoli, and A. Rettori, Europhys. Lett. 27, 159 (1994).

${ }^{7}$ P. Politi, A. Rettori, F. Hartmann-Boutron, and J. Villain, Phys. Rev. Lett. 75, 537 (1995).

${ }^{8}$ T. Lis, Acta Cryst. B 36, 2042 (1980).

${ }^{9}$ K. L. Taft, C. D. Delfs, G. C. Papaefthymiou, S. Foner, D. Gatteschi, and S. J. Lippard, J. Am. Chem. Soc. 116, 823 (1994).

${ }^{10}$ B. Barbara, W. Wernsdorfer, L. C. Sampaio, J. G. Park, C. Paulsen, M. A. Novak, R. Ferré, D. Mailly, R. Sessoli, A Caneschi, K. Hasselbach, A. Benoit, and L. Thomas, J. Magn. Magn. Mater. 140-144, 1825 (1995).

${ }^{11}$ C. Paulsen and J. G. Park, in Quantum Tunneling of Magnetization, edited by L. Gunther and B. Barbara (Kluwer, Dordrecht, 1995), pp. 189-207.

${ }^{12}$ M. A. Novak and R. Sessoli, in Quantum Tunneling of Magnetization, edited by L. Gunther and B. Barbara (Kluwer, Dordrecht, 1995), pp. 171188. 